\title{
ALFABETIZAÇÃO FINANCEIRA: UM ESTUDO POR MEIO DA APLICAÇÃO DA TEORIA DE RESPOSTA AO ITEM
}

FINANCIAL LITERACY: A STUDY USING

THE APPLICATION OF ITEM RESPONSE THEORY

Recebido em: 07/08/2015 Aprovado em: 16/10/2015 Avaliado pelo sistema double blind review Editora Científica: Cláudia de Salles Stadtlober DOI: 10.13058/raep.2016.v17n1.341

\section{JOÃO CARLOS HIPÓLITO BERNARDES DO NASCIMENTO joaohipolito@ufrj.br MARCELO ÁLVARO DA SILVAMACEDO JOSÉ RICARDO MAIA DE SIQUEIRA}

UNIVERSIDADE FEDERAL DO RIO DE JANEIRO

\section{JULIANA REIS BERNARDES}

UNIVERSIDADE ESTÁCIO DE SÁ

\begin{abstract}
RESUMO
O presente estudo teve por objetivo mensurar o nível de alfabetização financeira dos discentes do curso de Administração de empresas de uma Instituição de Ensino Superior (IES) Federal, sendo conduzida, para tal, uma survey com 307 alunos. Para análise dos dados, foi utilizada a Teoria de Resposta ao Item (TRI). A título de resultado, foram obtidos indícios que permitem concluir pelo baixo nível de alfabetização financeira e pela existência de um perfil conservador de investimento por parte dos discentes. Esse cenário, alinhado aos estudos empíricos anteriores realizados no país, mostra-se preocupante, dado o potencial de externalidades negativas decorrente de más decisões financeiras, sobretudo, relacionadas ao financiamento da casa própria e à preparação para a aposentadoria. O presente estudo contribui ao testar empiricamente, no contexto nacional, a utilização da TRI à estimação da alfabetização financeira, permitindo concluir que a TRI é uma opção metodológica importante na estimação desse traço latente, que possibilita a comparabilidade dos conhecimentos financeiros por meio de uma medida consistente e confiável, por meio de estudos, populações, realidades e programas distintos.

Palavras-chave: alfabetização financeira; habilidades latentes; Teoria de Resposta ao Item.
\end{abstract}

\footnotetext{
ABSTRACT

This study aimed to measure the level of financial literacy of Business Administration course students at a federal Higher Education Institution (HEI). To this end, a survey was conducted on 307 students. The Item Response Theory (IRT) was employed for data analysis and the findings support the conclusion that the students show a low level of financial literacy, as well as the existence of a conservative investment profile among students. This scenario, in line with previous empirical studies conducted in the Brazil, is worrying given the potential negative externalities resulting from poor financial decisions, especially those related to home financing and retirement preparations. This study contributes to the empirical evaluation, within the national context, of the use of IRT in estimating financial literacy, and shows that it is, indeed, an important methodological option in the estimation of this latent trait. Furthermore, this enables financial knowledge to be compared through consistent and reliable means, using studies, populations, realities and separate programs.

Keywords: financial literacy; latent skills; Item Response Theory.
} 


\section{INTRODUÇÃO}

A partir da primeira década dos anos 2000, foi notada, no Brasil, uma substancial melhoria dos indicadores de distribuição de renda decorrente, principalmente, da tríade: ciclo de crescimento econômico, políticas públicas de valorização do salário mínimo e de transferência de renda (BARBOSA, 20I2). Entretanto, surpreendentemente, não é notada uma evolução similar no tocante aos indicadores educacionais, a despeito da forte relação entre ambas variáveis, dado que, no médio e longo prazo, a distribuição eficiente de renda está intimamente relacionada à promoção da educação (FIELDS, I980; GREGORIO; LEE, 1999). Essa conjuntura tem levantado sérias preocupações acerca da segurança financeira da população, especialmente em relação àqueles que não têm as habilidades necessárias para tomar boas decisões financeiras, sendo nomeadamente as mais importantes: a aquisição e financiamento da casa própria e a preparação para a aposentadoria (HUNG; PARKER; YOONG, 2009).

Após o advento dos novos instrumentos financeiros criados no âmbito do Sistema de Financiamento Imobiliário (SFI), o nível de contratação de financiamentos habitacionais vem crescendo vertiginosamente desde o início de 2005, mesmo em meio ao aprofundamento da crise financeira internacional, em meados de 2008 (CAGNIN, 20I2). Segundo estimativas de Lima e Matias-Pereira (2014), no período de 2012 a 2030, a dinâmica demográfica incrementará em até cinco vezes a necessidade de financiamento observada no ano de 2003.

Relativo à previdência, o envelhecimento da população tem despertado a atenção dos governos para a necessidade de promoção de mecanismos de fomento ao incremento à idade média de aposentadoria visando minimizar o impacto sobre o setor público, bem como de mitigação dos constantes déficits da previdência social. Um exemplo dessa tendência é a Lei n ${ }^{\circ}$ 12.6I8/20I2 que instituiu o Regime de Previdência Complementar para os servidores públicos federais.

A despeito da iminente complexidade e relevância inerente a ambas as decisões supracitadas, a literatura revela que parte relevante da população 
mundial (DANES; HIRA, I987; VOLPE; CHEN; PAVLICKO, I996; CHEN; VOLPE, I998; BEAL; DELPACHITRA, 2003; MURPHY, 2005; KLAPPER; LUSARDI; PANOS, 20I3) e brasileira (VIEIRA; BATAGLIA; SEREIA, 2OII; ALVES; SILVA; BRESSAN, 2OII) apresenta baixos níveis de alfabetização financeira.

As primeiras definições de alfabetização financeira (financial literacy) caracterizavam-na como a capacidade de fazer julgamentos e tomar decisões efetivas sobre o uso e gestão do dinheiro. Desde então, vários autores têm contribuído no tocante às definições, entretanto, a essência tem sempre convergido nessa mesma direção. No presente estudo, foi adotado o conceito da OECD (20I2) que entende alfabetização financeira como a capacidade de usar o conhecimento e as habilidades para gerenciar os recursos financeiros de forma a proporcionar eficácia nas decisões financeiras.

Para mensuração da alfabetização financeira, tem-se utilizado desde testes univariados de médias e de frequência até, mais recentemente, a modelagens multivariadas. Entretanto, não é notada a existência de uma medida amplamente disseminada de alfabetização financeira desenvolvida por meio de análises psicométricas rigorosas o que acarreta, entre outras implicações, na baixa comparabilidade do desempenho dos participantes por meio de estudos, populações, realidades e programas distintos. Nesse aspecto, alinhada à recomendação de Knoll e Houts (2012), o presente estudo teve como objetivo mensurar o nível de alfabetização financeira dos discentes do curso de Administração de Empresas de uma Instituição de Ensino Superior (IES) Federal, utilizando, como técnica de análise dos dados, a Teoria de Resposta ao Item (TRI).

A TRI fundamenta-se em modelos matemáticos nos quais o cálculo da estimativa do traço latente leva em conta não só as respostas dadas pelos indivíduos, mas também, as propriedades dos itens que serviram para essa avaliação (EMBRETSON; REISE, 2000; REISE; AINSWORTH; HAVILAND, 2005).

Justifica-se a condução do presente estudo pela relevância da alfabetização financeira na atual conjuntura, sobretudo no cenário de existência de baixos níveis de alfabetização financeira por parte da população brasileira reportado pela literatura e, sobretudo, pela importância de testar a utilização da TRI para mensurar a alfabetização financeira tendo em vista que, segundo 
Knoll e Houts (20I2), essa modelagem possibilita a comparabilidade dos conhecimentos financeiros por meio de uma medida consistente e confiável, entre estudos, populações e programas distintos.

Além dessa Introdução, o estudo encontra-se segmentado em quatro tópicos. Inicialmente é realizada uma breve revisão da literatura acerca das técnicas utilizadas na mensuração do nível de alfabetização financeira no âmbito internacional, uma sucinta apresentação dos principais estudos nacionais sobre a temática, e uma explanação acerca do conceito, modelos, pressupostos e estimação dos parâmetros da TRI.

A seguir, são apresentados os aspectos metodológicos adotados no estudo, nomeadamente, o desenvolvimento e validação do instrumento de coleta de dados. Na sequência é realizada a análise e discussão dos dados e, por fim, são apresentadas as considerações finais com as devidas limitações e indicações de oportunidades para o desenvolvimento de futuras pesquisas. 


\section{MENSURAÇÃO DO NÍVEL DE ALFABETIZAÇÃO FINANCEIRA}

Os primeiros estudos sobre alfabetização financeira utilizavam a porcentagem média de acertos em testes específicos como técnica de mensuração. As observações eram agrupadas em três grupos: aqueles com rendimento acima de $80 \%$ apresentavam alto nível de conhecimento; na faixa de $60 \%$ a $79 \%$ nível médio de conhecimento e com acerto abaixo de $60 \%$ representava níveis baixos de conhecimento financeiro (DANES; HIRA, I987).

A partir da segunda metade da década de 90, os estudos começaram a adotar metodologias mais robustas à mensuração da alfabetização financeira. Chen e Volpe (1998), por exemplo, utilizaram Análise de Variância (ANOva) e Regressão logística. Nos anos 2000, é notada a utilização de Regressões Logísticas (CHEN; VOLPE, 2002; BEAL; DELPACHITRA, 2003; WORTHINGTON, 2004; MURPHY, 2005), Regressões Múltiplas (VOLPE; KOTEL; CHEN, 2002; JANG; HAHN; PARK, 20I4) e Dados em Painel (KLAPPER; LUSARDI; PANOS, 20I3).

Muito embora estudos com ferramentais mais simples ainda tenham sido conduzidos como, por exemplo, a utilização de Correlações e testes de médias por RMR (2003), nota-se a predominância de modelagens multivariadas à estimação da alfabetização funcional.

No entanto, a despeito dessa recente aderência aos métodos multivariados para a mensuração do nível de alfabetização financeira, existe uma relevante limitação na literatura sobre alfabetização financeira decorrente do fato do fenômeno alfabetização financeira não ser, necessariamente, dicotômico (alfabetizado ou não), mas um continuum.

O Quadro I, a seguir, apresenta um breve levantamento acerca das estratégias utilizadas para a mensuração da alfabetização financeira no âmbito internacional: 
MARCELO ÁLVARO DA SILVA MACEDO \& OUTROS

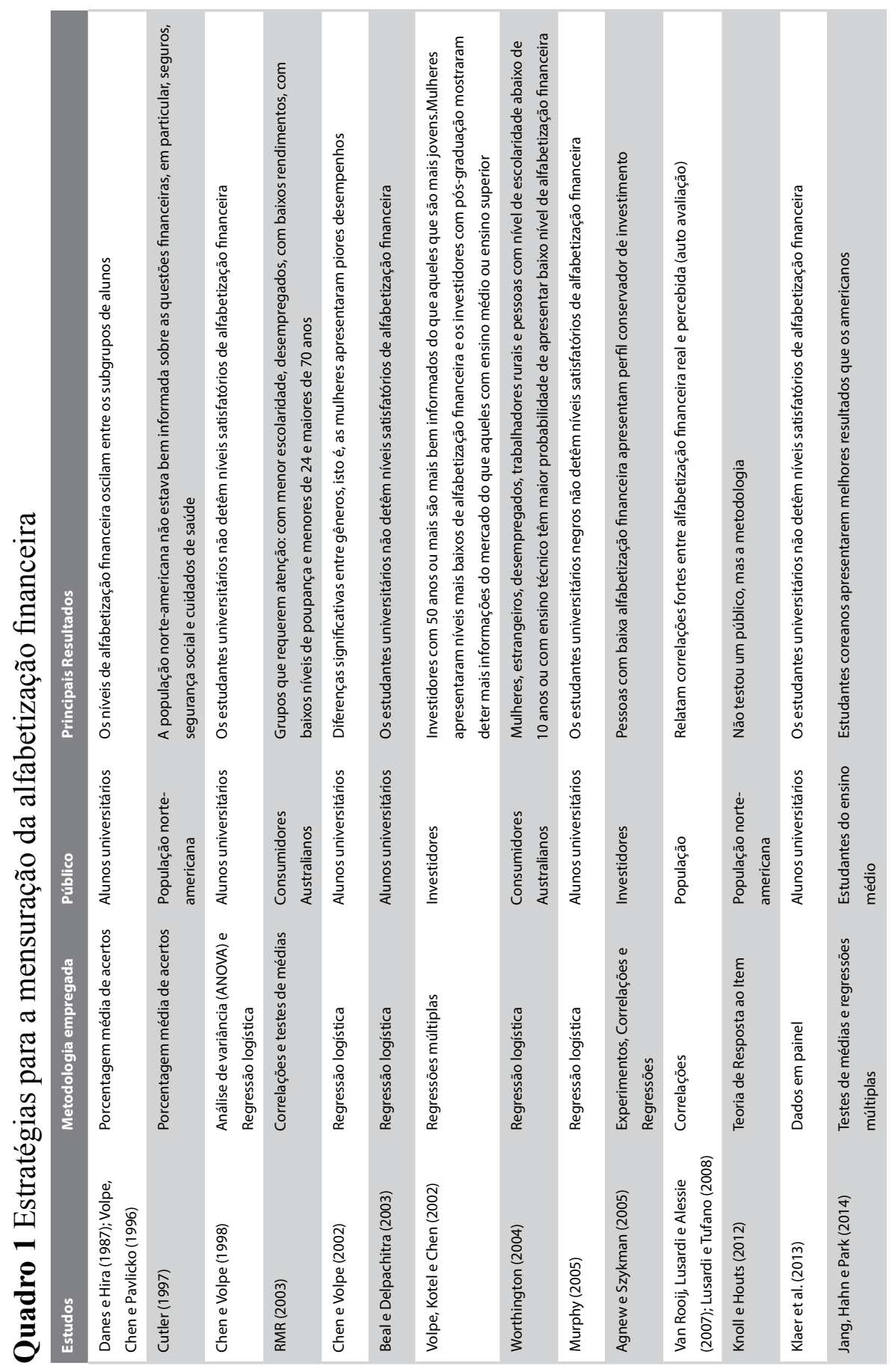


$\mathrm{Na}$ literatura nacional, corriqueiramente, utiliza-se testes de médias e de frequência para estimar o nível de alfabetização financeira. Lucci, Zerrenner e Verrone (2006), utilizando-se de estatística descritiva para analisar a influência da educação financeira nas decisões de consumo e investimento dos indivíduos, notaram que o conhecimento sobre finanças aprendidos na universidade influenciou positivamente na qualidade das decisões financeiras.Vieira, Bataglia e Sereia (20II) analisaram se a alfabetização financeira obtida junto aos cursos de graduação de Administração, Ciências Econômicas e Ciências Contábeis influenciam na atitude de consumo, poupança e investimento dos indivíduos. Para tal, por meio da utilização do teste estatístico não paramétrico, qui-quadrado de Pearson, aplicado a uma amostra de 303 alunos, os autores concluíram que a formação acadêmica contribui para a melhor tomada de decisões de consumo, investimento e poupança dos indivíduos.

Buscando confrontar o nível de alfabetização financeira de alunos de graduação em Ciências Contábeis de uma universidade privada do Rio de Janeiro e frente ao desempenho de alunos norte-americanos, Alves, Silva e Bressan (20II) utilizaram o teste $Z$ de proporções e obtiveram indícios empíricos de que $74 \%$ da amostra possui baixo nível de educação financeira, especialmente, quando confrontado com o desempenho de discentes estadunidenses.

Potrich, Vieira e Kirch (20I4), por meio da condução de uma survey com 1572 indivíduos, e utilizando-se da análise de conglomerados (clusters), concluíram que alunos do gênero masculino, sem dependentes, com maior grau de escolaridade e níveis elevados de renda própria e familiar apresentam maior propensão a possuir níveis elevados de alfabetização financeira.

Por fim, Potrich, Vieira e Ceretta (2013), utilizando-se de estatísticas descritivas, testes de diferença de média (teste t) e técnicas de análise multivariada de análise de variância (ANOva) e análise fatorial exploratória, notaram que os estudantes apresentam comportamento financeiro positivo, porém, não satisfatório, dado que não têm, de forma bem estabelecida, hábitos de poupar mensalmente e de manter uma reserva financeira para casos inesperados. 
É possível notar a inexistência de uma medida amplamente disseminada de alfabetização financeira desenvolvida por meio de análises psicométricas rigorosas. Nessa abordagem tradicional de mensuração de traços latentes, pautada na Teoria Clássica dos Testes (TCT), o desempenho é mensurado por meio da quantidade / percentual de itens corretos, o grau de dificuldade das questões é obtido restritamente pela proporção de erros e o grau de confiabilidade e o erro de medida são estimados globalmente, isto é, para o instrumento como um todo. Nessa abordagem, o poder discriminatório dos itens é obtido por meio de correlações bisserial e ponto-bisserial entre o item e o desempenho total no teste, ou ainda por meio da diferença no desempenho do item entre os $33 \%$ com maior nota total e os $33 \%$ com menor nota total baixa (HAYES, I992; PASQUALI, 2003; REISE; AINSWORTH; HAVILAND, 2005; RECKASE, 2009).

Nesse aspecto, a TCт apresenta como principal limitação o fato dos parâmetros do instrumento, isto é, informações do item, a confiabilidade da escala, validade e erros-padrões serem sensíveis à amostra trabalhada, o que demanda a necessidade de um grande número de participantes para validação do instrumento e, sobretudo, dificulta a obtenção de uma medida consistente e confiável, por meio de estudos, populações e realidades distintos (EMBRETSON, I996; REISE; AINSWORTH; HAVILAND, 2005; RECKASE, 2009).

Parte substancial dessas limitações é corrigida por meio da TRI que possibilita compor um instrumento de pesquisa considerando os parâmetros de dificuldade e de discriminação de cada item e, por consequência, do instrumento como um todo. Além disso, por meio do procedimento de equalização (equating) do teste, possibilita-se o desenvolvimento de vários instrumentos com níveis equivalentes de dificuldade e discriminação, possibilitando, assim, o acompanhamento longitudinal de turmas/indivíduos.

Logo, pode-se notar que a utilização da TRI para a mensuração da alfabetização financeira acarretará, potencialmente, em uma série de benefícios, sobretudo, no fomento à comparabilidade de desempenho de alunos longitudinalmente e por meio de estudos, populações, realidades e programas distintos. 


\section{TEORIA DE RESPOSTA AO ITEM}

Algumas variáveis, como por exemplo, a percepção da qualidade, a inteligibilidade e a compreensibilidade, entre outras, apresentam como principal característica o fato de não possibilitarem a observação direta, sendo, portanto, necessária a aferição com base na observação de variáveis secundárias relacionadas (VALLE, I999).

Esse tipo de variável, denominada como latente, pode ser estimada por meio do estabelecimento de relações entre o desempenho do indivíduo em determinado teste composto por itens e a variável de interesse (TAVARES, 200I), sendo a TRI a técnica predominante nas últimas décadas à estimação de habilidades latentes (ANDRADE; TAVARES; VALLE, 2000).

Pautada na premissa de que os itens que constituem o teste são os elementos centrais da análise, a TRI pode ser definida como um arcabouço de funções matemáticas que especifica o relacionamento de interação entre indivíduos e itens do teste (RECKASE, 2009). Nessa mesma perspectiva, Andrade, Tavares e Valle (2000) afirmam que a TRI é um conjunto de modelagens matemáticas que buscam estimar a probabilidade do respondente " $j$ ” apresentar a resposta correta ao item "i" de um determinado instrumento avaliativo em função dos parâmetros dos itens e do nível de proficiência (theta ou habilidade latente) do respondente.

Os valores de theta são estimados com base na distribuição normal das habilidades dos respondentes na sua padronização de forma similar aos escores $z$, cuja média é igual a zero $(0,00)$ e o desvio padrão é igual a um $(\mathrm{I}, 00)$. O nível de theta de cada indivíduo é então obtido por meio do somatório dos thetas parciais de cada item do instrumento avaliativo (ALEXANDRE et al., 200I).

Após a estimação de theta, são consideradas as probabilidades de o respondente acertar os demais itens do questionário, ou seja, dado determinado nível de traço latente, soma-se a probabilidade do individuo acertar o primeiro item, mais a probabilidade de acertar o segundo e assim por diante (MÜLLER, 2007).

Segundo Valle (I999, p. 3), no âmbito dos traços latentes que se pretende mensurar, os modelos podem assumir duas classificações: unidimensional quando o interesse limita-se em estimar um traço latente; e multidimensional: 
quando se busca examinar dois ou mais traços latentes simultaneamente. Entretanto, segundo Reckase (2009), toda a modelagem matemática da TRI foi fundamentada no modelo unidimensional, sendo esse o modelo mais utilizado na prática.

Existem vários modelos não lineares na TRI (um, dois ou três parâmetros), sendo o modelo logístico unidimensional de dois parâmetros (2PLM), desenvolvido por Lord em 1952 (LORD, I967, p. 383), o pioneiro. O modelo 2PLM é apresentado abaixo na Equação I (BAKER, 200I):

$$
\mathrm{P}\left(U_{i j}=1 \mid \theta_{j}\right)=\frac{1}{1+\mathrm{e}^{-a_{i}\left(\theta_{j}-\mathrm{b}_{j}\right)}}
$$

Onde:

$\mathrm{P}\left(U_{i j}=1 \mid \theta_{j}\right)$ é a probabilidade do respondente $j$ acertar o item $i$ considerando sua habilidade $\theta_{j}$;

$U_{i j}=$ é a resposta dada pelo respondente $j$ ao item $i$;

$\theta_{j}=$ habilidade ou traço latente do respondente $\mathrm{j}$;

$a_{i}=$ parâmetro de discriminação do item i;

$b_{i}=$ parâmetro de dificuldade do item $i$.

Existem dois pressupostos que devem ser observados a utilização de modelos unidimensionais da TRI: a unidimensionalidade do item e a independência local. A unidimensionalidade refere-se ao fato do teste avaliar apenas um único traço latente (ANDRADE; TAVARES; VALLE, 2000), enquanto a independência local pressupõe que, mantidas constantes as aptidões, com exceção do $\theta$ dominante, as respostas dos itens são independentes. Em outras palavras, o desempenho do indivíduo em determinado item não é afetado pelo desempenho nas demais questões do instrumento de coleta de dados (HAMBLETON; SWAMINATHAN; ROGERS, I99I).

A estimação dos parâmetros na TRI é realizada pelo Método da Máxima Verossimilhança por meio da utilização de algum processo interativo, como por exemplo, o algoritmo EM - Expectation Maximization (ANDRADE; TAVARES; VALLE, 2000). 
O algoritmo EM é uma ferramenta utilizada na estimação do estimador de máxima verossimilhança (EMv) de forma iterativa, inclusive suportando bases de dados com dados incompletos (CASELLA; BERGER, 20II).

Quando comparada à abordagem da TCT, a TRI apresenta como principais vantagens: a estimação e controle dos parâmetros de dificuldade, discriminação e probabilidade de acerto casual (no caso do modelo logístico unidimensional de três parâmetros - 3PLM); a invariância dos parâmetros independentemente da amostra estudada; o cálculo do grau de confiabilidade e o erro padrão de cada item; e a equalização do teste que possibilita acompanhamentos longitudinais de turmas/indivíduos (HAYES, I992; EMBRETSON, I996; ANDRADE; TAVARES; VALLE, 2000; REISE, AINSWORTH; HAVILAND, 2005; RECKASE, 2009). 


\section{METODOLOGIA}

Dado que o instrumento de coleta de dados utilizado por Knoll e Houts (2012) apresenta 20 itens, em decorrência da disponibilidade de tempo dos alunos, optou-se pelo desenvolvimento de um questionário mais sucinto visando criar incentivos à maior adesão à pesquisa, porém, conservando as características do instrumento seminal. Os itens da versão final do instrumento demandam conhecimento acerca de volatilidade, ativos financeiros, planejamento financeiro, finanças pessoais e instrumentos de política monetária, como a SELIC.

Como apresentado anteriormente, o objetivo do presente estudo não é a validação do instrumento desenvolvido por Knoll e Houts (20I2) ao contexto brasileiro, mas sim, a avaliação da plausibilidade da utilização da TRI à estimação da alfabetização financeira no âmbito nacional. Assim, esforços futuros devem ser direcionados à construção de um instrumento mais amplo, englobando itens alinhados às particularidades do contexto brasileiro via TRI.

Inicialmente foi realizado um pré-teste aplicando o questionário a uma turma de 36 alunos, visando analisar a adequação dos itens e o dimensionamento do tempo necessário para a participação. Concluída essa etapa inicial, foi dado prosseguimento à aplicação às demais turmas.

Da amostra inicial de 322 alunos, I5 questionários foram descartados em decorrência da existência de missing, assim, foram compreendidas 307 observações. Dada à existência de disciplinas específicas de Finanças na grade curricular do curso de graduação em Administração, espera-se notar evidências da existência de níveis intermediários a avançado de alfabetização financeira.

Inicialmente buscou-se avaliar a consistência interna do instrumento de pesquisa. Para tal, dada a natureza binária dos dados, foi utilizado o método de Kuder-Richardson. O valor de o,87I, obtido na análise, permitiu concluir pela existência de um bom nível de consistência interna (GEORGE; MALLERY, 2003).

Concluída a análise da consistência interna, seguiu-se à validação do instrumento de coleta de dados. Este procedimento foi realizado por meio 
da Análise Fatorial Exploratória, por meio do software FACTOR v9.3, software gratuito desenvolvido pelos professores Urbano Lorenzo-Seva e Pere Joan Ferrando, da Universitat Rovira $i$ Virgili (Tarragona, Espanha).

Mais uma vez, em decorrência da natureza dicotômica dos dados, a análise fatorial confirmatória foi realizada utilizando correlações policóricas (polychoric correlations). O índice de 0,735 para a estatística Kaiser-MeyerOlkim (кмо), o valor Qui Quadrado do teste de Bartlett de 293,9, significante a o,ooo e a variância total explicada de 6I,6I\% após a extração de um único fator, permitiram concluir pela existência de apenas um fator captado pelo questionário. Muito embora inexista unanimidade na definição de um único critério objetivo, conclui-se pela unidimensionalidade quando a variância total explicada é superior a 50\% (HAIR JÚNIOR et al., 20IO). Ao se confirmar a unidimensionalidade, consequentemente obtém-se a independência local (HAMBLETON; SWAMINATHAN; ROGERS, I99I).

O GFI (goodness-of-fit index ou índice de qualidade do ajuste), coeficiente de determinação geral para modelos de equações estruturais análogo ao $\mathrm{R}^{2}$ da regressão múltipla, indica a proporção de variância-covariância explicada pelo modelo. $\mathrm{O}$ valor obtido de 0,99 permite concluir elevada adequação ao modelo (TANAKA, 1993). Outra medida importante, o valor obtido à raiz da média dos quadrados dos erros de aproximação (RMSEA) de o,075 - abaixo de 0,08 , teto para ser considerado desejável (THOMPSON, 2004) - indica quão bem o modelo se ajusta à população (não apenas à amostra utilizada para a estimação).

Confirmado os pressupostos da TRI, a análise teve continuidade. Alinhado a Knoll e Houts (2012), o nível de alfabetização financeira dos discentes foi mensurado com base no modelo logístico unidimensional para itens dicotômicos com dois parâmetros, com as respostas sendo categorizadas como correta ou incorreta, sendo as respostas "não sei”, quando disponíveis, tratadas como incorretas.

Os parâmetros do modelo 2PLM da TRI foram estimados usando o algoritmo EM por meio do software IRTPRo. A opção pela estimação baseada no algoritmo EM também se encontra alinhada ao estudo e Knoll e Houts (2012). 


\section{ANÁLISE DOS DADOS}

\section{MENSURAÇÃO DO NÍVEL DE ALFABETIZAÇÃO FINANCEIRA}

Como apresentado anteriormente, os parâmetros dos itens propostos para avaliar o nível de alfabetização financeira foram estimados com base no modelo logístico unidimensional para itens dicotômicos com dois parâmetros. Os valores estimados dos parâmetros $(a)$ e $(b)$ e seus respectivos erros padrões são apresentados na Tabela I.

Tabela I Estimativas dos parâmetros e erros padrões dos itens

\begin{tabular}{|lllll|}
\hline Item & Parâmetro (a) & Erro padrão (a) & Parâmetro (b) & Erro padrão (b) \\
\hline 1 & 0,85 & 0,20 & $-1,30$ & 0,29 \\
\hline 2 & 2,10 & 0,42 & $-0,56$ & 0,10 \\
\hline 3 & 2,33 & 0,45 & $-0,12$ & 0,09 \\
\hline 4 & 2,22 & 0,42 & 0,02 & 0,09 \\
\hline 5 & 2,07 & 0,38 & 0,04 & 0,09 \\
\hline Média & $\mathbf{1 , 9 1}$ & $\mathbf{0 , 3 7}$ & $\mathbf{- 0 , 3 8}$ & $\mathbf{0 , 1 3}$ \\
\hline Desvio & $\mathbf{0 , 6 0}$ & - & $\mathbf{0 , 5 7}$ & - \\
\hline
\end{tabular}

O parâmetro (a) mensura o nível de discriminação do item, isto é, aplicado ao presente estudo, o parâmetro distingue discentes com níveis distintos de alfabetização financeira. Este parâmetro reflete a inclinação da curva do modelo logístico, sendo que, quanto maior o parâmetro, mais robusta é a capacidade de discriminação dos alunos com níveis diferenciados de alfabetização financeira.

Muito embora possa assumir valores entre - $\infty$ a $+\infty$, não é comum a ocorrência de valores negativos (ANDRADE; TAVARES; VALLE, 2000; BAKER, 200I), sendo usualmente utilizados itens com valores orbitando no intervalo de o a 2 (HAMBleton; SWAMinATHAN; Rogers, I99I). Na Tabela I, é possível notar que os itens 2, 3, 4 e 5 ostentam maior poder discriminatório, sendo que a capacidade média de segregação do teste é positiva em I,9I, com desvio padrão de 0,60 , resultado alinhado ao preconizado pela literatura. 
O parâmetro de dificuldade $(b)$, que apresenta valores esperados de -3 a +3 , corresponde ao valor de theta (habilidade latente) e, no caso do presente estudo, capta o nível de alfabetização financeira. Segundo Hambleton, Swaminathan e Rogers (199I), se $(b)<\theta_{j}$, é mais provável que o respondente acerte o item, caso $(b)>\theta_{j}$ provavelmente o indivíduo erre o item e, por fim, se $(b)=\theta_{j}$ sugere dificuldade mediana, exigindo uma habilidade média (percentil de 50) de alfabetização financeira para acertar o item. Em outras palavras, quanto maior o valor do parâmetro, menor a probabilidade de um aluno com baixo nível de alfabetização financeira acertar o item.

Novamente analisando a Tabela I, pode-se concluir pela existência de itens com variados níveis de dificuldade no questionário o que, segundo Harraway e Barker (2005), é desejável. O item I demanda de baixo a mediano nível de alfabetização financeira para o acerto, enquanto os demais itens captam nível intermediário. Dado que o valor médio do parâmetro (b) é de -0,38 pode-se concluir que o instrumento apresenta nível satisfatório para captar o nível de alfabetização financeira.

A análise do parâmetro (b) de cada item evidencia o nível de alfabetização financeira que o item em específico é capaz de captar, conforme evidenciado em ordem decrescente na Tabela 2.

Tabela 2 Itens conforme nível de captação da alfabetização financeira

\begin{tabular}{|ll|}
\hline Item & Parâmetro (b) \\
\hline 5 & 0,04 \\
\hline 4 & 0,02 \\
\hline 3 & $-0,12$ \\
\hline 2 & $-0,56$ \\
\hline 1 & $-1,30$ \\
\hline
\end{tabular}

A curva de informação do teste, apresentada na Figura I, evidencia a capacidade do instrumento captar distintos níveis de alfabetização financeira, compreendendo o somatório de informação de todos os itens em cada região da escala de habilidade latente. 
Figura I Curva de informação do instrumento

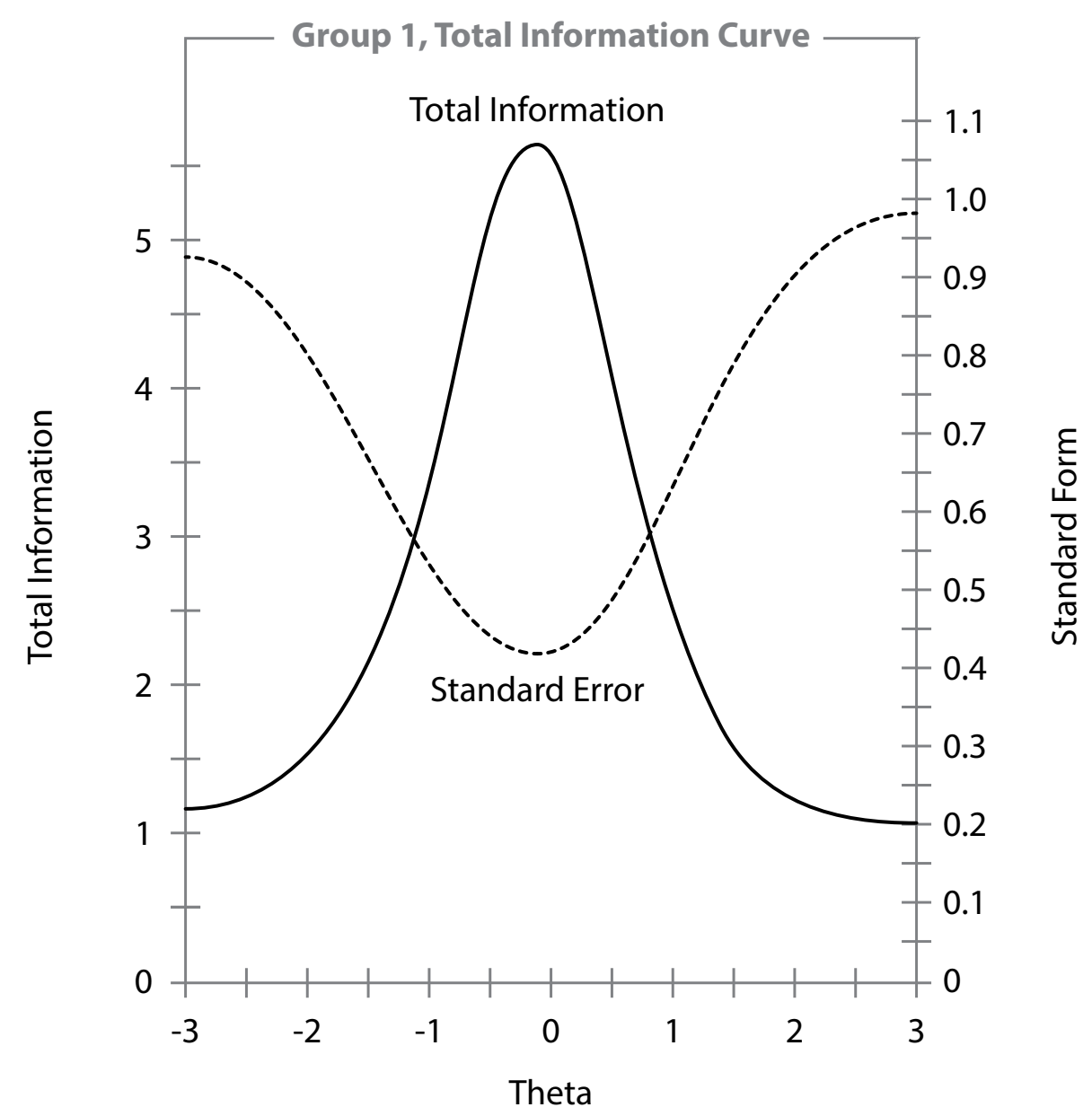

A função informação do teste evidencia a região da escala de habilidade latente em que existe maior precisão do instrumento, isto é, para qual nível de alfabetização financeira é capturada maior informação. Analisando a Figura I, é possível concluir que o instrumento capta um maior volume de informação entre os níveis de conhecimento financeiro de $-\mathrm{I}, 5$ a $+\mathrm{I}, 5$, aproximadamente. Na Tabela 3, são apresentadas as estatísticas descritivas do nível de alfabetização financeira dos discentes. 
Tabela 3 Estatísticas descritivas do nível de alfabetização financeira dos discentes

\begin{tabular}{|lc|}
\hline Médlia & 0,0002 \\
\hline Erro padrão & 0,0478 \\
\hline Mediana & $-0,0560$ \\
\hline Moda & 1,0990 \\
\hline Desvio padrão & 0,8381 \\
\hline Variância da amostra & 0,7024 \\
\hline Curtose & $-1,2682$ \\
\hline Assimetria & 0,0048 \\
\hline Intervalo & 2,5010 \\
\hline Mínimo & $-1,4020$ \\
\hline Máximo & 1,0990 \\
\hline Contagem & 307 \\
\hline
\end{tabular}

Considerando a moda de I,099, a mediana (-0,0560) e média (0,0002), podese concluir pela existência de uma distribuição assimétrica positiva ou à direita (assimetria de 0,0048 ), ou seja, a maior parte dos alunos apresenta desempenho inferior à média. Adicionalmente, o valor de -I,2682 para curtose indica uma distribuição platicúrtica, isto é, as observações encontram-se relativamente dispersas em torno da média.

Analisando a distância entre o valor mínimo (-I,4020) e máximo (I,0990), pode-se notar a existência de importante amplitude dos dados, fato este corroborado pelo desvio padrão de 0,838I. O nível de alfabetização financeira dos discentes é apresentado na Figura 2. 
Figura 2 Distribuição dos discentes conforme nível de alfabetização financeira

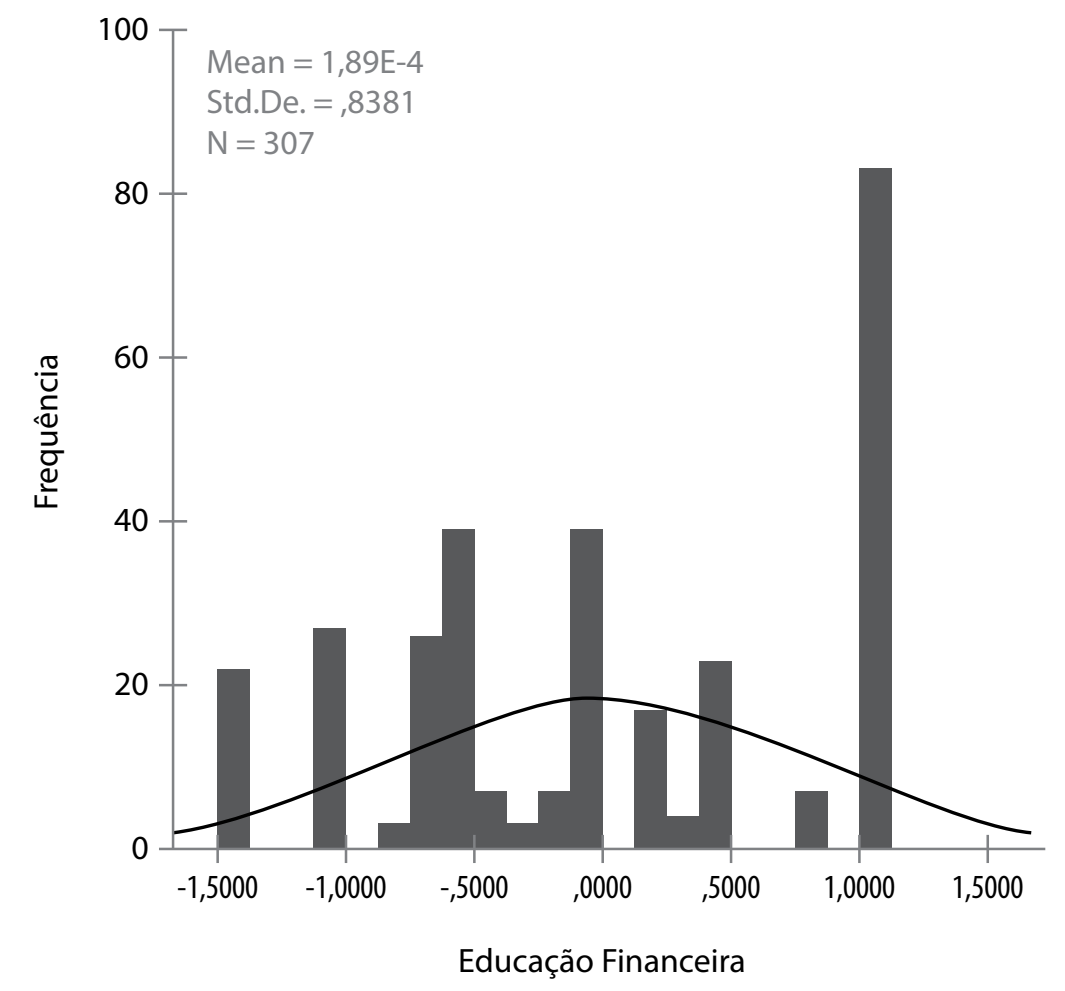

Com base na distribuição dos discentes conforme nível de alfabetização financeira é possível notar que 83 alunos (27\%) apresentaram nível razoável de alfabetização financeira, isto é, com theta de I a I,I numa escala que orbita de $-3 \mathrm{a}+3$. Parte dos discentes, $5 \mathrm{I}$ ou $16 \%$ aproximadamente, ostentou desempenho de o, I a o, 8 , enquanto a grande maioria 173 ou $56,35 \%$ apresentou baixo nível de conhecimento financeiro, ou seja, theta oscilantes de -I,5 a o, sendo que 72 alunos $(23,45 \%)$ apresentaram thetas no intervalo de -I,5 a -0,7. Nesse ponto, pode-se concluir pela existência de baixo nível de alfabetização financeira por parte dos graduandos em Administração de Empresas.

Visando analisar a existência de diferença de desempenho dos alunos nos 8 (oito) períodos do curso, foi realizado o teste não paramétrico KruskalWallis para amostras independentes. Considerando a significância de o,oo obtida no teste, a hipótese nula de que todas as populações possuem 
funções de distribuição iguais pôde ser rejeitada, ou seja, existem diferenças estatisticamente significativas em pelo menos dois dos períodos analisados, como pode ser observado na Figura 3.

Figura 3 Desempenho médio por período

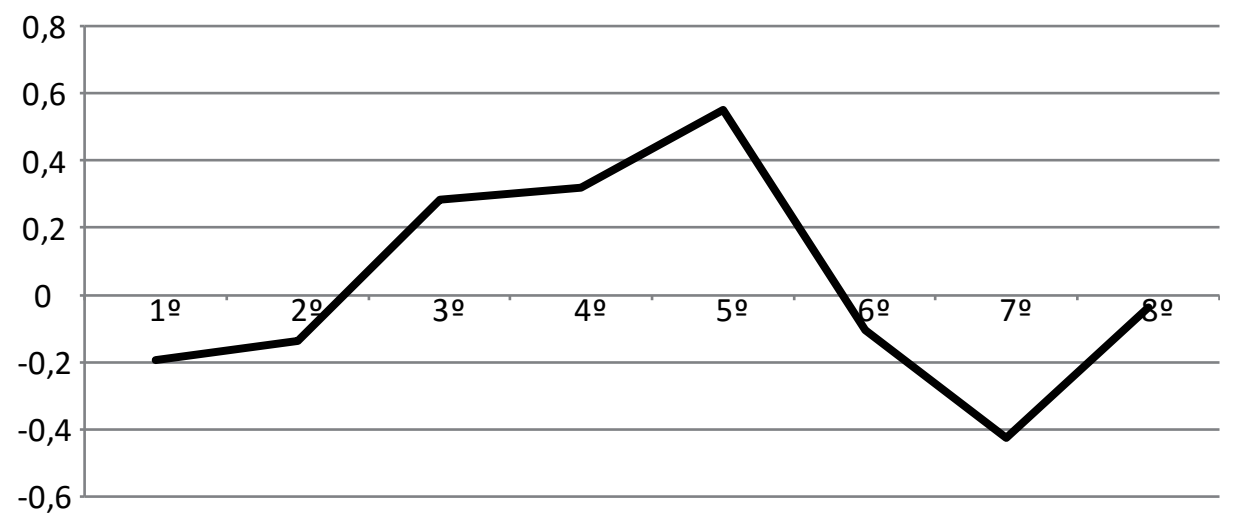

É possível notar, na Figura 3, que a tendência ascendente é revertida a partir do $6^{\circ}$ período, tendo o pior desempenho no $7^{\circ}$ período. Essa realidade pode ser decorrente da concentração das disciplinas de Finanças no meio do curso, do fato dos alunos terem estudado apenas com o intuito de serem aprovados na disciplina e, principalmente, da percepção, por parte dos alunos, de que os conteúdos discutidos na disciplina não têm aplicação imediata no atual momento da vida (estudos).

O resultado notado no $8^{\circ}$ período, quando confrontado com o apresentado no $\mathrm{I}^{\circ}$ período não foi estatisticamente distinto, conforme a significância de 0,35I do teste Mann-Whitney. Como não houve um acompanhamento longitudinal com a mesma turma, não é possível afirmar pela inexistência de evolução do nível de alfabetização financeira dentro da graduação, mas apenas que não foi notada a existência de diferenças significativas entre ingressantes e concluintes. Esses resultados corroboram parcialmente com os achados de Vieira, Bataglia e Sereia (20II) que notaram que, em algumas questões, o desempenho dos alunos ingressantes e concluintes não apresentou diferenças significativas. 
Esses achados mostram-se ainda mais relevantes quando se pondera que esses discentes tão logo estarão no mercado de trabalho, diariamente, exercendo decisões de captação e aplicação de recursos financeiros e, potencialmente, gerando externalidades negativas para as empresas, stakeholders e a si próprios. A despeito da existência de disciplinas específicas de Finanças na grade curricular, o desempenho dos discentes mostrou-se pouco satisfatório, levantando questionamentos acerca do eventual desempenho de discentes de cursos que não dispõem de disciplinas correlatas na grade curricular.

No que diz respeito ao desempenho da TRI para a mensuração da alfabetização financeira, pode-se notar que a TRI mostrou-se uma opção metodológica importante na estimação desse traço latente, possibilitando a comparabilidade dos conhecimentos financeiros por meio de uma medida consistente e confiável, entre estudos, populações, realidades e programas distintos. Nesse aspecto, esforços devem ser direcionados à construção de um instrumento mais amplo, englobando a proposição de itens mais alinhados às particularidades do contexto brasileiro via TRI.

Além das vantagens já apresentadas, a aplicação da TRI permite aos pesquisadores desenvolverem instrumentos compostos por subconjuntos de questões particularmente relevantes a propósitos específicos sem a necessidade de administrar todo o conjunto de itens do instrumento (tendo em vista a validação prévio dos itens), reduzindo, assim, uma série de problemas relacionados à aplicação de questionários como, por exemplo, a saturação dos respondentes, tempo de aplicação e baixa taxa de retorno. Assim, torna-se plausível desenvolver instrumentos com itens especificamente relacionados, por exemplo, a financiamentos, com itens estreitamente relacionados à questão de pesquisa.

Por fim, ressalta-se que no eventual interesse de grupos e subtemas específicos, por exemplo, jovens adultos e suas estratégias de planejamento da aposentadoria, é possível, por meio da equalização do teste, desenvolver testes específicos por meio da inclusão ou exclusão de itens (KNOLL; HOUTS, 20I2).

\section{PERFIL E ESTRATÉGIAS DE INVESTIMENTOS}

Concluída a mensuração do nível de alfabetização financeira dos graduandos 
e a consequente avaliação da adequabilidade da TRI para mensuração da alfabetização financeira, a seguir são reportadas informações incrementais coletadas. Inicialmente notou-se que apenas $27,69 \%$ ou 85 alunos costumam poupar parte do orçamento mensal com um objetivo específico. Para esse grupo de poupadores, 52,87\% (46 respondentes) poupam sem nenhum motivo específico (28,74\% afirmaram não ter objetivo específico, 12,64\% escolheram a opção "Outros” e II,49\% não responderam). Ademais, 22,99\% (20 alunos) sinalizaram preocupação com a aposentadoria, I7,24\% (I5 discentes) justificaram investir em educação e 3,45\% (três alunos) planejam viajar ou investir em saúde.

Esse cenário é especialmente preocupante dada a atual conjuntura de recorrentes déficits nas contas da previdência social e a iminente necessidade da realização de previdência complementar. Outro dado importante ocorre pela baixa poupança com o objetivo de contratar serviços de saúde.

Quando questionados acerca da estratégia utilizada, isto é, a linha de crédito geralmente utilizada para fechar as contas no final do mês, 36,48\% afirmou não utilizar nenhuma linha de financiamento o que pode ser explicado pelo financiamento por parte dos pais. Cerca de $16 \%$ utilizam crédito pessoal, $7,5 \%$ crédito consignado e $3,58 \%$ optou por não responder.

No tocante às linhas disponibilizadas pelo mercado, $24,76 \%$ dos alunos confessaram utilizar o limite do cheque especial e II,40\% o refinanciamento da fatura do cartão de crédito, opções que apresentam como principal característica a exorbitante taxa de juros praticada, o que vai de encontro ao preconizado pela cartilha da alfabetização financeira e reforça a conclusão de baixo nível de alfabetização financeira nos discentes.

Notou-se ainda que, aproximadamente, 6I\% da amostra, I88 alunos, investem na caderneta de poupança, resultado que permite concluir que, de uma forma geral, os discentes apresentam perfil de investimento conservador. Por fim, questionados acerca da motivação para o não investimento em ações, entre outras opções, cerca de $45 \%$ alegou falta de conhecimento e $18 \%$ afirmou preferir opções de investimentos menos voláteis, o que corrobora, respectivamente, o baixo nível de alfabetização financeira e o perfil conservador de investimento dos alunos. 


\section{CONSIDERAÇÕES FINAIS}

Cidadãos com baixos níveis de alfabetização financeira são, inconscientemente, mais propensos a cometerem erros financeiros, menos propensos a se envolverem em práticas financeiras recomendadas e incapazes de lidar com choques econômicos repentinos (KLAPPER; LUSARDI; PANOS, 20I3). Esse cenário torna-se ainda mais relevante no contexto brasileiro, onde se pratica uma das mais altas taxas de juros do mundo.

Nesse aspecto, o presente estudo teve por objetivo mensurar o nível de alfabetização financeira dos discentes do curso de Administração de empresas de uma Instituição de Ensino Superior (IEs) Federal. A despeito da existência de disciplinas específicas de Finanças na grade curricular do curso de graduação em Administração, por meio da utilização da TRI, foram notados indícios que permitem concluir pela existência de baixo nível de alfabetização financeira por parte dos discentes.

Embora tenha sido notada uma evolução ascendente importante, após o $6^{\circ}$ período a tendência foi invertida, acarretando em níveis similares de alfabetização financeira entre ingressantes e concluintes. Mesmo não tendo sido realizado um estudo longitudinal, é possível formular o insight de que não houve na amostra uma evolução significativa na graduação em Administração.

Baixos níveis de alfabetização financeira, entre outros efeitos, acarretam em baixa participação nos mercados financeiros (investimentos) e alta utilização de fontes informais de empréstimo, que acarretam em altas taxas de jurOS (KLAPPER; LUSARDI; PANOS, 20I3).

Acerca das estratégias de investimentos dos graduandos, notou-se que estes apresentam perfil conservador e que poucos (cerca de 37\%) efetuam investimentos, sendo que apenas 9,77\% sinalizaram preocupação com a aposentadoria e 4,23\% poupam para investir em saúde.

Esses achados encontram-se alinhados ao preconizado pela literatura prévia, o que evidencia a relevância da problemática dentro do ensino superior, sobretudo, nos cursos da área de gestão, como é o caso específico do curso de Administração de empresas. Muito embora os resultados 
obtidos sejam similares à literatura prévia, o presente estudo contribui ao testar empiricamente, no contexto nacional, a utilização da TRI à estimação da alfabetização financeira.

Os resultados obtidos permitem concluir que a TRI é uma opção metodológica importante na estimação desse traço latente, possibilitando a comparabilidade dos conhecimentos financeiros por meio de uma medida consistente e confiável, entre estudos, populações, realidades e programas distintos.

A título de limitações, cita-se a impossibilidade de generalizar os resultados alcançados, tendo em vista que esses são limitados à amostra analisada, bem como a não apresentação dos itens de forma randômica no questionário o que, eventualmente, penaliza os últimos itens do instrumento em decorrência, dentre outros fatores, da saturação e cansaço.

Outra limitação surge na impossibilidade de explicar o motivo para o baixo nível de alfabetização financeira nos alunos concluintes do curso. Uma abordagem quanti-quali contribuiria à mitigação dessa fragilidade. Cita-se ainda como limitação o fato do instrumento de coleta de dados aplicado não ser equivalente ao utilizado por Knoll e Houts (2012), o que impossibilitou a confrontação dos resultados alcançados.

A exemplo de Vieira, Bataglia e Sereia (20II), o presente estudo também não contemplou variáveis relevantes à alfabetização funcional, como por exemplo, a experiência prática, fatores esses que devem ser compreendidos em futuras investigações. Como apresentado anteriormente, esforços podem ser direcionados à construção de um instrumento mais amplo, englobando a proposição de itens mais alinhados às particularidades do contexto brasileiro via TRI.

Sugere-se ainda, acerca das oportunidades para a condução de futuras pesquisas, visando detectar a evolução do nível de alfabetização financeira, efetuar um estudo longitudinal, acompanhando turmas do início até a conclusão da graduação. Outra oportunidade surge na condução de estudos que confrontem o resultado decorrente da estimação via TRI frente à adoção de uma abordagem qualitativa visando captar as eventuais motivações da ocorrência de baixos níveis de alfabetização financeira mesmo após os discentes terem cursado as disciplinas de Finanças na graduação. 
Por fim, a condução de estudos semelhantes em outros cursos, sobretudo naqueles que não apresentam disciplinas específicas de Finanças na grade curricular, bem como confrontar os resultados do curso de Administração frente a outros cursos correlatos, como por exemplo, Ciências Contábeis, Ciências Atuariais e Economia visando detectar a existência de diferenças significativas. Por fim, nota-se uma oportunidade relevante no tocante à detecção dos determinantes à alfabetização financeira. 


\section{REFERÊNCIAS}

AGNEW, J. R.; SZYKMAN, L. R. Asset allocation and information overload: The influence of information display, asset choice, and investor experience. Journal of Behavioral Finance, v. 6, n.1, p. 57-70, 2005.

ALEXANDRE, J. W. C.; ANDRADE, D.F.; VASCONCELOS, A. P.; ARAUJO, A. M. S. Uma proposta de análise de um construto para a medição dos fatores críticos da gestão pela qualidade por meio da TRI. Gestão \& Produção, v. 9, n. 2, p. 129-141, 2001.

ALVES, R. A.; SILVA, J. S.; BRESSAN, A. A. Alfabetização financeira de Discentes em Ciências Contábeis: Diagnóstico e Comparação com Universitários Norte-Americanos. Congresso Nacional de Administração e Ciências Contábeis - AdCont, Anais... Rio de Janeiro - RJ, Rio de Janeiro, 2, 2011.

ANDRADE, D. F.; TAVARES, H. R.; VALLE, R. C. Teoria de Resposta ao Item: conceitos e aplicações. ABE - Associação Brasileira de Estatística, 4 SINAPE, 2000.

BAKER, FRANK B. The Basics of Item Response Theory. New York: ERIC Clearinghouse on Assessment and Evaluation, 2001.

BALBIM JUNIOR, A.; BORNIA, A. C. Proposta de um instrumento de medida para avaliar a satisfação de clientes de bancos utilizando a Teoria da Resposta ao Item. Gestão \& Produção, v. 18, n. 3, p. 541-554, 2011.

BARBOSA, L. M. L. H. A evolução recente da distribuição de renda brasileira sob a ótica das estruturas sócio-ocupacionais. 2012. 163 f. Dissertação (Mestrado em Economia) - Programa de Pós Graduação em Economia. Instituto de Economia da Universidade Federal do Rio de Janeiro, Rio de Janeiro, 2012.

BEAL, D.; DELPACHITRA, S. Financial literacy among Australian university students. Economic Papers: a Journal of Applied Economics and Policy, v. 22, n. 1, p. 65-78, 2003.

CAGNIN, R. F. A evolução do financiamento habitacional no Brasil entre 2005 e 2011 e o desempenho dos novos instrumentos financeiros. Boletim de Economia, v. 11, n.1, p. 15-32, 2012.

CASELLA, G.; BERGER, R. L. Inferência estatística. Tradução da $2^{\mathrm{a}}$ edição norteamericana. São Paulo: Centage Learning, 2011.

CHEN, H.; VOLPE, R. P. An Analysis of Personal Financial Literacy Among College Students. Financial Services Review, v. 7, n. 2, p. 107-128, 1998.

CHEN, H.; VOLPE, R. P. Gender Differences in Personal Financial Literacy Among College Students. Financial Services Review, v. 11, n.3, p. 289-307, 2002.

CUTLER, N.E. The False Alarms and Blaring Sirens of Financial Literacy: Middle-Agers' Knowledge of Retirement Income, Health Finance and Long-Term Care, Generations, v. 21, n. 2, p. 34-40, 1997.

DANES, S. M.; HIRA, T. K. Money management knowledge of college students. The Journal of Student Financial Aid, v. 17, n.1, p. 4-16, 1987. 
EMBRETSON, S. E. The new rules of measurement. Psychological Assessment, v. 8, n. 4, p. 341-349, 1996.

EMBRETSON, S. E.; REISE, S. P. Item Response Theory for psychologists. Hillsdale, NJ: Lawrence Erlbaum, 2000.

FIELDS, G. S. Education and income distribution in developing countries: A review of the literature. In T. King (Ed.), Education and income: A background study for world development (231-315). Washington, DC: The World Bank, 1980.

FLETCHER, F. R. A Teoria de Respostas ao Item: Medidas Invariantes do desempenho escolar. Ensaio, v. 1, n. 2, p. $21-27,1994$.

GEORGE, D.; MALLERY, P. SPSS for Windows step by step: A simple guide and reference. 11.0 update. 4.ed. Boston: Allyn \& Bacon, 2003.

GREGORIO, J. DE; LEE, J.-W. Education and Income Distribution: New Evidence from Cross-country Data. Documentos de Trabajo, v. 55, Centro de Economía Aplicada, Universidad de Chile, 1999.

HAIR JÚNIOR, J.; BLACK, W. C.; BABIN, B. J.; ANDERSON, R. E. Multivariate data analysis.7.ed. Upper Saddle River: Prentice Hall, 2010.

HAMBLETON, R. K.; SWAMINATHAN, H.; ROGERS, H. J. Fundamental of Item Response Theory. London: Sage Publications, 1991.

HAYES, B. E. Measuring customer satisfaction: development and use of questionnaires. Milwaukee, Wisconsin: ASQC Quality Press, 1992.

HUNG, A. A.; PARKER, A. M.; YOONG, J. K. Defining and Measuring Financial Literacy. Working Paper, 2009.

JANG, K.; HAHN, J.; PARK, H. J. Comparison of financial literacy between Korean and U.S. high school students. International Review of Economics Education, v. 16, n.1, p. 22-38, 2014.

KLAPPER, L.; LUSARDI, A.; PANOS, G.A. Financial literacy and its consequences: Evidence from Russia during the financial crisis. Journal of Banking \& Finance, v. 37, p. 3904-3923, 2013.

KNOLL, M. A. Z.; HOUTS, C. R. The Financial Knowledge Scale: An Alication of Item Response Theory to the Assessment of Financial Literacy. Journal of Consumer Affairs, v. 46, n. 3, p. 381-410, 2012.

LIMA, D. V.; MATIAS-PEREIRA, J. A dinâmica demográfica e a sustentabilidade do regime geral de previdência social brasileiro. RAP - Revista de Administração Pública, v. 48, n. 4, p. 847-868, 2014.

LORD, F. M. An analysis of the Verbal Scholastic Aptitude Test using Birnbaum's three parameter logistic model. Princeton, NJ: Educational Testing Service, 1967.

LUCCI, C. R.; ZERRENNER, S. A.; VERRONE, M. A. G.; SANTOS, S. C. A Influência da Educação Financeira nas Decisões de Consumo e Investimento dos Indivíduos. Anais... Seminários em Administração (SEMEAD). São Paulo, 2006. 
LUSARDI, A.; MITCHELL, O.S. Financial literacy and retirement planning: New evidence from the RAND American Life Panel. MRRC Working Paper 2007-157, 2007.

LUSARDI, A.; PANOS, P. Debt Literacy, Financial Experiences and Overindebtedness. NBER Working Papers 14808, National Bureau of Economic Research, Inc, 2013.

MÜLLER, S. I. M. G. Sistema integrado de avaliação com aplicação na engenharia. 2007. Tese de Doutorado em Métodos Numéricos em Engenharia, Universidade Federal do Paraná, Curitiba/PR, 2007.

MURPHY, A. J. Money, Money, Money: An Exploratory Study on the Financial Literacy of Black College Students. College Student Journal, v. 39, n. 3, p. 478-488, 2005.

NOCTOR, M.; STONEY, S.; STRADLING, R. Financial Literacy: A Discussion of Concepts and Competences of Financial Literacy and Oortunities for its Introduction into Young People's Learning. Report prepared for the National Westminster Bank, National Foundation for Education Research, London, 1992.

ORGANISATION FOR ECONOMIC CO-OPERATION AND DEVELOPMENT (OECD). High-Level Principles on National Strategies for Financial Education, OECD Publishing, 2012.

PASQUALI, L. Psicometria: Teoria dos testes na psicologia e na educação. Petrópolis/RJ: Vozes, 2003.

POTRICH, A. C. G.; VIEIRA, K. M.; CERETTA, P. S. Nível de alfabetização financeira dos estudantes universitários: afinal, o que é relevante? Revista Eletrônica de Ciência Administrativa, v. 12, n. 3, p. 314-333, 2013.

POTRICH, A. C. G.; VIEIRA, K. M.; KIRCH, G. Determinantes da Alfabetização Financeira: Proposição de um Modelo e Análise da Influência das Variáveis Socioeconômicas e Demográficas. Anais... XXXVIII Encontro da Associação Nacional de Pós-Graduação e Pesquisa em Administração - ANPAD, Rio de Janeiro/RJ, 2014.

RAY MORGAN RESEARCH (RMR). ANZ Survey of Adult Financial Literacy in Australia: Final Report, May 2003. Melbourne: Ray Morgan Research, 2003.

RECKASE, M. D. Statistics for Social and Behavioral Sciences: Multidimensional Item Response Theory. Springer: New York, 2009.

REISE, S.; AINSWORTH, A.; HAVILAND, M. Item Response Theory. Current Directions in Psychological Science, v. 14, n. 2, p. 95-101, 2005.

TANAKA, J. S. Multifaceted conceptions of fit in structural equation models. In: BOLLEN, K.A.; LONG, J.S. (Eds.). Testing structural equation models (10-39). Newbury Park, CA: Sage, 1993.

TAVARES, H. R. Teoria da Resposta ao Item para dados longitudinais. 2001. Tese de Doutorado em Estatística, Universidade de São Paulo, São Paulo/SP, 2001.

THOMPSON, B. Exploratory and Confirmatory Factor Analysis. Washington. DC: American Psychological Association, 2004. 
VALLE, R C. Teoria da Resposta ao Item. 1999. 218 f. Dissertação Dissertação (Mestrado em Estatística) - Universidade de São Paulo, São Paulo/SP, 1999.

VAN ROOIJ, M.; LUSARDI, A.; ALESSIE, R. Financial literacy and stock market participation. MRRC Working Paper, 2007.

VIEIRA, S. F. A.; BATAGLIA, R. T. M.; SEREIA, V. J. Educação financeira e decisões de consumo, investimento e poupança: uma análise dos alunos de uma universidade pública do norte do Paraná. Revista de Administração da UNIMEP, v. 9, n. 3, p. 62-86, 2011.

VITÓRIA, F.; ALMEIDA, L. S.; PRIMI, R. Unidimensionalidade em testes psicológicos: conceito, estratégias e dificuldades na sua avaliação. Revista de Psicologia da Vetor Editora, v. 7, n. 1, p. 1-7, 2007.

VOLPE, R. P.; CHEN, H.; PAVLICKO, J. J. Personal investment literacy among college students: A survey. Financial Practice and Education, v. 6, n. 2, p. 86-94, 1996.

VOLPE, R. P.; KOTEL, J. E.; CHEN, H. A survey of investment literacy among online investors. Financial Counseling and Planning, v. 13, n. 1, p. 1-16, 2002.

WORTHINGTON, A. C. The Distribution of Financial Literacy in Australia, Discussion Papers in Economics, Finance and International Competitiveness, Queensland University of Technology, Brisbane, Discussion Paper 185, 2004. 


\section{DADOS DOS AUTORES}

\section{JOÃO CARLOS HIPÓLITO BERNARDES DO \\ NASCIMENTO` joaohipolito@ufrj.br}

Mestre em Ciências Contábeis pela FUCAPE Business School

Instituição de vinculação: Universidade Federal do Rio de Janeiro

Rio de Janeiro/RJ - Brasil

Áreas de interesse em pesquisa: Ensino e Pesquisa em Administração e Contabilidade, Governança Corporativa e Gestão Pública.

*Quadra Z 1 , Lote 31 Topol Casa Nova/BA 47300-000

\section{MARCELO ÁLVARO DA SILVA MACEDO malvaro.facc.ufrj@gmail.com}

Doutor em Engenharia de Produção pela COPPE/UFRJ

Instituição de vinculação: Universidade Federal do Rio de Janeiro

Rio de Janeiro/RJ - Brasil

Áreas de interesse em pesquisa: Contabilidade, Finanças, Métodos Quantitativos

Aplicados à Contabilidade.

JOSÉ RICARDO MAIA DE SIQUEIRA jrms@facc.ufrj.br

Doutor em Engenharia de Produção pela COPPE/UFRJ

Instituição de vinculação: Universidade Federal do Rio de Janeiro

Rio de Janeiro/RJ - Brasil

Áreas de interesse em pesquisa: Relatórios de Sustentabilidade, Responsabilidade Social, Ensino e Pesquisa em Administração e Contabilidade.

\section{JULIANA REIS BERNARDES julianareiszd@gmail.com}

Licenciada em Letras pela Universidade de Pernambuco

Instituição de vinculação: Universidade Estácio de Sá

Rio de Janeiro/RJ - Brasil

Áreas de interesse em pesquisa: Consumo, Marketing, Ensino e Pesquisa em

Administração e Contabilidade. 